\title{
ESTUDO DA FOTOCATÁLISE HETEROGÊNEA SOBRE Ti/TiO 2 NA DESCOLORAÇÃO DE CORANTES SINTÉTICOS
}

\author{
Thiago N. M. Cervantes, Dimas A. M. Zaia e Henrique de Santana* \\ Departamento de Química, Universidade Estadual de Londrina, 86051-990 Londrina - PR, Brasil
}

Recebido em 29/4/09; aceito em 26/5/09; publicado na web em 28/10/09

\begin{abstract}
A HETEROGENEOUS PHOTOCATALYSIS STUDY OF DECOLORIZATION OF SYNTHETIC DYES ON Ti/TiO 2 . The main goal of this paper was to study the degradation of synthetic dyes using photoelectrocatalytic properties of particulate films of $\mathrm{TiO}_{2}$ supported on plates of titanium and stimulated by UV-Vis radiation. The dyes decolorizations were measured using spectrophotometric methods to verify which the conditions on $\mathrm{Ti} / \mathrm{TiO}_{2}$ electrode was the best for the photoelectrodegradation of them. The results showed that decolorization rates were higher than $90 \%$ during a period of $270 \mathrm{~min}$. FT-IR spectroscopy showed that intermediate substances were formed after the decolorization and $\mathrm{N}=\mathrm{N}$ group/aromatic structures were preserved independently of the specific structure of the dyes.
\end{abstract}

Keywords: heterogeneous photocatalysis; titanium dioxide; synthetic dyes.

\section{INTRODUÇÃO}

Uma grande quantidade de poluentes químicos, provenientes das mais diversas atividades, vem causando muita preocupação na comunidade científica, principalmente no que estes agentes podem acarretar ao meio ambiente, onde a contaminação do mesmo tem sido apontada como um dos maiores problemas da sociedade moderna. ${ }^{1}$

Com isto, novas normas e legislações cada vez mais rígidas têm sido adotadas em todo o mundo com a finalidade de minimizar o impacto dos poluentes ao meio ambiente. No Brasil, esta forma de controle tem se mostrado insuficiente e este fato, somado à ausência de processos de tratamento adequados, é uma realidade preocupante. Segundo dados da CETESB, a geração de resíduos perigosos atinge 820.000 toneladas por ano, sendo que 376.000 são geradas apenas na região metropolitana de São Paulo. ${ }^{1}$

As indústrias de couro, em conjunto com as indústrias têxteis, são exemplos de atividades produtivas responsáveis pela contaminação de águas naturais. Isso se deve ao fato que estas atividades produzem uma grande quantidade de efluentes provenientes de tingimentos com corantes reativos que não são considerados tóxicos, mas podem sofrer uma degradação parcial biológica ou provocada por outras formas de tratamento, fazendo com que as moléculas parcialmente degradadas se liguem a outras moléculas, levando à formação de compostos muito mais tóxicos que os corantes originais. ${ }^{2}$ Este problema tem sido especialmente encontrado em corantes contendo grupos cromóforos azo, caracterizados pela ligação $-\mathrm{N}=\mathrm{N}-$ ligados a sistemas aromáticos, cuja classe pertence a maior parte dos corantes reativos. ${ }^{3}$

Estes corantes sintéticos lançados num curso d'água em direção aos rios, sem tratamentos prévios eficientes, são capazes de colorir parcialmente um manancial, estando na água em quantidades ínfimas. Como resultado, a luz solar deixa de atingir as plantas aquáticas, o que impede que elas promovam a fotossíntese e se reproduzam adequadamente. Como consequência, os peixes têm a oferta de alimentos e oxigênio reduzida. ${ }^{4}$

Os corantes também podem atingir uma estação de captação e tratamento de água, comprometendo assim o abastecimento populacional, além de contaminar o solo próximo ao manancial. Nesse aspecto, surge um outro problema: algumas bactérias presentes no solo ajudam a degradar o corante, porém podem produzir compostos

*e-mail: hensan@uel.br carcinogênicos. ${ }^{4}$ Estes, ao atingirem a água e o solo, representam um grande problema para as culturas agrícolas, uma das bases alimentares de homens e animais. Diante desta grande ameaça, faz-se necessário o desenvolvimento de novos métodos de tratamento destes efluentes que garantam baixo nível de contaminantes.

Neste sentido, a fotocatálise pode ser uma forma eficiente e versátil de controle da poluição aquosa, modificando, degradando ou mineralizando compostos orgânicos através de processos onde o único reagente envolvido é o elétron. ${ }^{5-7}$ Temos os chamados "Processos Oxidativos Avançados" (POA), que vêm atraindo grande interesse por serem mais sustentáveis em longo prazo. Os POA dividem-se em sistemas heterogêneos e homogêneos, onde radicais são gerados com ou sem irradiação ultravioleta. Entre estes, podem-se citar como heterogêneos os processos que envolvem a utilização de semicondutores (fotocatálise heterogênea). ${ }^{8}$

Dentre os óxidos metálicos semicondutores temos $\mathrm{TiO}_{2}, \mathrm{ZnO}$, $\mathrm{WO}_{3}, \mathrm{SrO}_{3}$ e $\mathrm{Fe}_{2} \mathrm{O}_{3}$, onde o $\mathrm{TiO}_{2}$ é o mais utilizado devido, principalmente, à sua não toxidade, alta atividade fotoquímica, baixo custo, estabilidade em sistemas aquosos e estabilidade química em uma ampla faixa de $\mathrm{pH} .{ }^{9,10}$ Entre os diferentes fabricantes, o $\mathrm{TiO}_{2}$ fabricado pela Degussa, $\mathrm{TiO}_{2} \mathrm{P}_{2} 5^{\circledR}$ (70\% anatase e $30 \%$ rutilo), é o mais comumente utilizado devido sua alta fotoatividade quando comparada com outras fontes. Isto se deve à sua alta área superficial, em torno de $50 \mathrm{~m}^{2} / \mathrm{g}$, e à sua microestrutura cristalina complexa resultante de seu método de preparação, que promove melhor separação de cargas inibindo sua recombinação. ${ }^{11} \mathrm{~A}$ recombinação elétron/lacuna é apontada como o principal limitante para o rendimento total do processo. Algumas tentativas para minimizar tal recombinação têm sido estudadas, tal como a incorporação de metais à sua estrutura cristalina ou à sua superfície. ${ }^{10}$

\section{Princípio da fotocatálise heterogênea}

O princípio da fotocatálise heterogênea envolve a ativação de um semicondutor (geralmente $\mathrm{TiO}_{2}$ ) por luz solar ou artificial. Um semicondutor é caracterizado por bandas de valência e bandas de condução, sendo a região entre elas chamada de bandgap. ${ }^{2}$ Quando um semicondutor é irradiado com radiação ultravioleta, a uma energia maior que sua energia de bandgap, são gerados elétrons $\left(\mathrm{e}_{C B}{ }^{-}\right)$ na banda de condução e lacunas $\left(\mathrm{h}_{\mathrm{VB}}{ }^{+}\right)$na banda de valência. Os elétrons fotogerados podem reagir tanto com o corante quanto com 
elétrons aceptores, por exemplo, moléculas de oxigênio adsorvidas na superfície do semicondutor ou dissolvidas na água, gerando um superóxido $\mathrm{O}_{2} \cdot$. As lacunas fotogeradas podem oxidar a molécula orgânica formando $\mathrm{R}^{+}$, ou reagir com $\mathrm{OH}^{-}$ou $\mathrm{H}_{2} \mathrm{O}$ oxidando estas espécies a $\mathrm{OH}$. Outras espécies altamente oxidantes (por exemplo, radicais de água oxigenada) são responsáveis pela fotodecomposição heterogênea com semicondutores nos substratos orgânicos, tais como corantes.

$\mathrm{O}$ radical $\mathrm{OH}$ resultante é um agente oxidante muito forte e pode oxidar a maioria dos corantes. Deve ser notado que a oxidação fotossensibilizada pode ocorrer, a qual se constitui da excitação por luz visível (não UV) do corante, e sua subsequente interação com o semicondutor. $^{12-14}$

Quando é utilizada luz solar ou uma fonte de luz artificial, é possível que ambos os mecanismos (foto-oxidação e fotossensibilização) ocorram, tornando difícil distinguir se o mecanismo de oxidação fotocatalítica é superior ao de oxidação fotossensibilizada.

Um fator que diminui a eficiência do processo fotoquímico é a recombinação do elétron com lacuna, antes do mesmo interagir com o corante. Uma forma de evitar esta recombinação é utilizar a irradiação UV com aplicação simultânea de um potencial externo anódico sobre um eletrodo semicondutor, forçando assim a separação das espécies carregadas. A esta combinação dos métodos eletroquímico e fotoquímico é dada a denominação de fotoeletrocatálise. ${ }^{7}$

As degradações que utilizam suspensões de $\mathrm{TiO}_{2}$ são experimentalmente muito trabalhosas, pois levam à necessidade de sucessivas filtrações para separação dos produtos da reação e o óxido. A fixação do catalisador num suporte estacionário torna a técnica mais prática, eliminando a etapa de filtração. ${ }^{8}$ Desta forma, podem ser confeccionados dispositivos do tipo reator tubular de vidro $^{15} \mathrm{e}$ membranas cerâmicas ${ }^{16}$ adequados para criar um sistema fechado de tratamento de águas.

Alguns parâmetros estudados separadamente, com objetivo de aumentar a eficiência do processo catalítico, têm sido de grande importância: intensidade de radiação ultravioleta, ${ }^{17}$ tempo de radiação, ${ }^{18}$ $\mathrm{TiO}_{2}$ dopado com diferentes íons metálicos de transição, ${ }^{19}$ natureza e espessura do filme de $\mathrm{TiO}_{2},{ }^{20}$ potencial aplicado ${ }^{21}$ e influência do pH. ${ }^{21,22}$

Para se entender os fatores que governam a fotodegradação eletrocatalítica de corantes sobre eletrodos semicondutores são necessários estudos mais sistemáticos dos parâmetros que influenciam o processo.

Neste artigo foi utilizado o mesmo azocorante para o qual foram descritos anteriormente os fatores fotoeletrocatalíticos que afetaram a eficiência da decomposição de um corante utilizado na indústria do couro sobre o sistema: substrato semicondutor (ITO)/ $/ \mathrm{TiO}_{2} / \mathrm{KCl} .^{23}$ Neste trabalho foi observado por FT-IR uma frequência em 1405 $\mathrm{cm}^{-1}$, atribuída ao estiramento $(\mathrm{N}=\mathrm{N})$ de um composto intermediário formado por decomposição do azocorante.

O objetivo deste trabalho foi fixar as condições experimentais no experimento fotoeletrocatalítico nas novas condições implementadas neste trabalho utilizando o azocorante (corante 2). Feito isso, aplicaram-se as mesmas condições sobre a degradação de quatro corantes têxteis (remazois). Considerou-se como positivo o fato do corante 2 dispor de uma estrutura química diferente dos corantes da família Remazol, que entre si também diferem em suas estruturas. As soluções após degradação foram analisadas por espectrofotometria UV-Vis e, depois de liofilizadas, por FT-IR. Os resultados indicaram o completo desaparecimento das bandas atribuídas ao grupo azo conjugado à parte cromófora e procurou-se discutir os prováveis produtos intermediários formados, que se assemelham ao produto formado pela degradação do corante 2, apesar da estrutura química divergir das dos demais.

\section{PARTE EXPERIMENTAL}

Em todos os experimentos foram utilizados reagentes P.A. e água Milli-Q. Os azocorantes estudados foram: corante 2, utilizado como recebido da Empresa Clariant e os remazois: Alaranjado Remazol 3 R, Amarelo Ouro Remazol RNL, Preto Remazol B e Remazol Red RB-133, utilizados como recebidos da Empresa DyStar. Os corantes aqui utilizados foram produzidos conforme as normas técnicas para este produto.

\section{Preparação dos eletrodos}

Os eletrodos de titânio foram inicialmente polidos utilizando-se palha de aço em água. Em seguida, deixaram-se os eletrodos em um béquer contendo acetona (Synth) e sonicou-se no ultrassom por 15 min (para limpeza). As placas de titânio foram secas utilizando estufa aquecida a $100^{\circ} \mathrm{C}$ por 5 min e pesadas em uma balança analítica. ${ }^{24}$

\section{Preparação do filme particulado de $\mathrm{TiO}_{2}$ sobre $\mathrm{Ti}$}

Com auxílio de uma micropipeta, foram medidos $0,39 \mathrm{~mL}$ de $\mathrm{NH}_{4} \mathrm{OH}$ (Synth) e 0,34 mL de $\mathrm{H}_{2} \mathrm{O}_{2}$ (Synth), sendo, em seguida, transferidos para um balão volumétrico de $10 \mathrm{~mL}$, completado com água Milli-Q. À esta solução foi adicionado $0,7 \mathrm{~g}$ de $\mathrm{TiO}_{2}$ (Degussa, $\mathrm{d}=3,8 \mathrm{~g} / \mathrm{cm}^{3}$ ) em um béquer de $50 \mathrm{~mL}$. Feito isso, a solução foi aquecida a $55^{\circ} \mathrm{C}$ sob agitação magnética por $\sim 2 \mathrm{~h}$ até a formação do gel. Para aumentar a fluidez do gel, foram adicionadas 3 gotas de Triton X-100 (Mallinckrodt) e 7 gotas de acetilacetona (Aldrich). Durante o processo de aquecimento, o gel apresentou uma consistência levemente viscosa e uma coloração amarelada. ${ }^{24}$

Antes de a emulsão ser depositada sobre a placa de Ti, foi previamente medida a massa da placa. Após a formação da emulsão, o gel foi cuidadosamente aplicado sobre as placas de Ti (Gruppo De Nora) com o auxílio de um bastão de vidro, sendo depositada uma única camada sobre uma área de $1,2 \mathrm{~cm}^{2}$. Os filmes com o depósito de $\mathrm{TiO}_{2}$ foram levados à estufa a $100^{\circ} \mathrm{C}$ por $10 \mathrm{~min}$, visando a evaporação do excesso de solvente, e após esta secagem foram mantidos na mufla a $500{ }^{\circ} \mathrm{C}$ por $2 \mathrm{~h}$.

Após a sinterização, deixou-se os filmes alcançarem a temperatura ambiente e foram obtidas as massas de $\mathrm{TiO}_{2}$ depositadas sobre as placas de Ti. Por meio da relação volume do filme/área do filme, analogamente ao relatado por Lipp e Pletcher, foi feita uma estimativa da espessura dos filmes de $\mathrm{TiO}_{2}$ sobre as placas de $\mathrm{Ti}^{25}$

\section{Caracterização por difração de raios-X do filme particulado de $\mathrm{TiO}_{2}$ sobre $\mathrm{Ti}$}

As medidas de difração de raios-X (DRX) foram conduzidas em um difratômetro convencional (Shimadzu - 6000), operando na geometria convencional $\theta-2 \theta$. A radiação utilizada foi a $\mathrm{K} \alpha$ do cobre $(\lambda=0,15418 \mathrm{~nm})$

\section{Câmara de irradiação}

Os experimentos de fotodegradação do azocorante foram realizados em uma câmara de irradiação, conforme descrito em de Santana, Bonancêa e Takashima. ${ }^{8}$ Somente foi substituído o potenciostato por uma fonte de tensão (c.c.).

\section{Curvas de calibração}

Para as concentrações de $0,01 \mathrm{mg} / \mathrm{mL}$ de azocorantes em $\mathrm{KCl}$ $10^{-1} \mathrm{~mol} \mathrm{~L}^{-1}$, ajustando as soluções para o $\mathrm{pH} 3,35$, foram obtidos 
os máximos de absorção na região do visível. A partir deles, foram determinadas as curvas de calibração numa faixa de variação de concentração de 0,02 a $0,0001 \mathrm{mg} / \mathrm{mL}$, com uma boa correlação observada (valor médio de $\left.\left(\mathrm{R}^{2}\right)=0,9980 \pm 0,001\right)$.

\section{Descoloração do azocorante}

A medida da descoloração consistiu em verificar a diminuição na coloração da solução contendo o azocorante, efetuando-se a leitura das absorvâncias das amostras de cada experimento fotoeletrocatalítico e determinou-se contra a curva de calibração a porcentagem de descoloração.

\section{Caracterização das estruturas moleculares por espectroscopia de absorção FT-IR}

O monitoramento da fotodegradação das estruturas moleculares dos corantes remazois foi realizado através de espectros FT-IR, obtidos antes e após as degradações. Para tal, as soluções foram liofilizadas e, posteriormente, prensadas em pastilhas de $\mathrm{KBr}$.

\section{RESULTADOS E DISCUSSÃO}

\section{Caracterização do filme particulado de $\mathrm{TiO}_{2}$ sobre $\mathrm{Ti}$}

Experimentos realizados com a técnica de difração de raios-X da placa de Ti e a placa preparada de $\mathrm{Ti} / \mathrm{TiO}_{2}$ fizeram-se necessários, considerando que o material de partida foi o $\mathrm{TiO}_{2}\left(\mathrm{P} 25^{\oplus}\right)$, com a composição de $70 \%$ anatase e $30 \%$ rutilo. Este material foi tratado quimicamente para a formação do gel e, posteriormente, foi aplicado sobre as placas de Ti, para a formação do eletrodo de $\mathrm{Ti} / \mathrm{TiO}_{2}$. Dos difratogramas foram obtidos picos de reflexão bem definidos em 25 e 48 graus, atribuídos à forma cristalina anatase do $\mathrm{TiO}_{2}$, e em 28, 42 e 55 graus, atribuídos à forma cristalina rutila do $\mathrm{TiO}_{2}$ (em relação aos picos observados provenientes da placa de Ti). ${ }^{26,27}$ Desta forma, constatou-se que mesmo após o tratamento químico de formação de gel para aplicação sobre as placas de titânio foram mantidas as duas formas cristalinas do dióxido de titânio. Considerando este resultado, foram utilizados nos experimentos como excitação do semicondutor a lâmpada de vapor de mercúrio com bulbo. Esta lâmpada emite intensamente entre 300 a $546 \mathrm{~nm}$, produzindo assim maiores quantidades de pares elétron-buraco no $\mathrm{TiO}_{2}$, consequentemente proporcionando uma maior eficiência na degradação de compostos sobre a superfície do eletrodo. ${ }^{23}$

\section{Quantificação da descoloração}

O azocorante, denominado corante 2 , foi utilizado inicialmente para o estudo dos diferentes parâmetros experimentais envolvidos no processo fotoeletrocatalítico. O intuito da utilização deste poluente foi definir as condições experimentais ideais para a maior eficiência no sistema $\mathrm{Ti} / \mathrm{TiO}_{2}$, com o objetivo de degradar outros corantes aplicados à indústria têxtil.

O corante 2 foi o mesmo composto utilizado em experimentos com filmes particulados de $\mathrm{TiO}_{2}$ sobre substratos de ITO (óxido de estanho dopado com índio), onde foi utilizado um potenciostato para aplicação do potencial sobre o eletrodo de trabalho de ITO/TiO, obtendo-se porcentagens máximas de descoloração de $81 \% .{ }^{23}$ Neste trabalho foram mantidas constantes a área do eletrodo, quantidade de solução, concentração do corante e o pH, sendo modificado o tempo de irradiação (de 8 para 4,5 h), massa/espessura do filme de $\mathrm{TiO}_{2} \mathrm{e}$ a forma de aplicação da ddp, agora entre os eletrodos de $\mathrm{Ti}^{\mathrm{T}} / \mathrm{TiO}_{2} \mathrm{e}$ Ti, utilizando uma fonte de tensão (c.c.). Desta forma, pretendeu-se buscar uma forma mais prática para construção de um fotorreator, adequado as exigências práticas das indústrias.

Com o intuito de ser feita uma análise das variáveis experimentais estudadas neste trabalho, seguem algumas constatações observadas em estudos anteriores. Após alguns estudos preliminares, o valor de $\mathrm{pH}$ foi mantido em 3,35, devido ao fato de ter um valor intermediário entre o valor de $\mathrm{pH}$ mais ácido, $\mathrm{pH} 1,0$ e o valor $\mathrm{pH}$ do ponto de carga zero $\left(\mathrm{pH}_{\mathrm{pcz}}\right)$ do $\mathrm{TiO}_{2}$, que é de $6,3 .{ }^{23} \mathrm{O}$ valor de $\mathrm{pH}$ em 3,35 foi também conveniente pela facilidade de ajuste da série de soluções utilizadas neste meio. A escolha do tempo de $4,5 \mathrm{~h}$ foi devido ao fato que os corantes da família Remazol degradam mais facilmente no sistema estudado. Dados preliminares demonstraram que após 2 $\mathrm{h}$ de irradiação o corante Preto Remazol B descolore completamente, entre 2,5 a 3 h de irradiação ocorre a descoloração dos corantes Alaranjado Remazol 3 R e Amarelo Ouro Remazol RNL, mas somente após $4 \mathrm{~h}$ de irradiação se verifica o mesmo efeito para o Remazol Red RB-133. Considerou-se para estes compostos que após 4,5 h de irradiação a degradação esteja favorecendo tanto a perda da cor como a formação e permanência de compostos intermediários. Estudos dirigidos sobre o efeito da espessura do filme de $\mathrm{TiO}_{2}$ sobre placas de titânio ${ }^{24}$ demonstraram que ocorre uma diferença de descoloração pouco pronunciada utilizando filmes de diferentes espessuras; mas em filmes com espessuras críticas, abaixo de $2 \mu \mathrm{m}$, a descoloração torna-se mais efetiva em potencial de $1,5 \mathrm{~V}$ comparado com os potenciais de 5 e $15 \mathrm{~V}$. Já para o filme com espessura acima de $2 \mu \mathrm{m}$ a descoloração se torna mais efetiva entre os potenciais de 1,5 a $5 \mathrm{~V}$ comparados com os potenciais entre 5 a $15 \mathrm{~V}$.

$\mathrm{Na}$ Tabela 1 são apresentados os resultados obtidos da porcentagem de descoloração do azocorante 2 em função do potencial aplicado sobre o eletrodo de $\mathrm{Ti} / \mathrm{TiO}_{2}$. Durante os experimentos, foram mantidos constantes a concentração do eletrólito, $\mathrm{pH}$, massa, espessura do filme de $\mathrm{TiO}_{2}$ e temperatura. Foram realizadas três medidas em cada condição para a validação dos resultados, sendo as condições experimentais mantidas em condições estatisticamente iguais.

Tabela 1. Descoloração do azocorante $2(0,01 \mathrm{mg} / \mathrm{mL})$ em $\mathrm{KCl} 0,1$ mol L-1 em pH 3,35. Período de irradiação: 4,5 h

\begin{tabular}{ccccc}
\hline $\mathrm{m}(\mathrm{mg})$ & $\mathrm{e}(\mu \mathrm{m})$ & $\mathrm{T}\left({ }^{\circ} \mathrm{C}\right)$ & $\mathrm{E}(\mathrm{V})$ & $\mathrm{D}(\%)$ \\
\hline $2,33 \pm 0,23$ & $5,56 \pm 0,55$ & $30,1 \pm 0,85$ & 5 & $* 96,40 \pm 1,15$ \\
$(3)$ & $(3)$ & & & $(3)$ \\
$2,23 \pm 0,32$ & $5,31 \pm 0,77$ & $30,1 \pm 0,85$ & 1 & $* 83,20 \pm 1,80$ \\
$(3)$ & $(3)$ & & & $(3)$ \\
\hline
\end{tabular}

Obs:. $\mathrm{m}=$ massa de $\mathrm{TiO}_{2}$ depositado sobre $\mathrm{Ti} ; \mathrm{e}=$ espessura dos filmes; $\mathrm{T}=$ temperatura; $\mathrm{E}=$ diferença de potencial aplicado entre os eletrodos de $\mathrm{Ti} / \mathrm{TiO}_{2}$ e Ti; D = descoloração. * valores estatisticamente diferentes.

Os resultados demonstraram que a melhor descoloração ocorreu para o potencial aplicado de $5 \mathrm{~V}$, sendo os dados obtidos estatisticamente diferentes $(\mathrm{p}<0,05)$, evidenciando que o potencial aplicado sobre os eletrodos de $\mathrm{Ti} / \mathrm{TiO}_{2}$ se constitui em um parâmetro que interfere diretamente na eficiência fotocatalítica. Estes resultados foram considerados básicos para o estudo da descoloração dos corantes remazois.

Definidas as condições experimentais do método, utilizando o corante 2, passou-se a trabalhar com corantes provenientes da indústria têxtil. Os corantes utilizados nesse estudo foram o Alaranjado Remazol 3 R, Amarelo Ouro Remazol RNL, Preto Remazol B e Remazol Red RB-133. Esses corantes foram submetidos às mesmas condições experimentais descritas anteriormente, exceto na variação do potencial - em vez de serem utilizados dois potenciais diferentes (5 e $1 \mathrm{~V}$ ) - foi utilizado apenas o potencial de maior eficiência na fotoeletrocatálise $(5 \mathrm{~V})$. 
Na Tabela 2 são apresentados os resultados obtidos da porcentagem de descoloração dos corantes remazois em função do potencial aplicado sobre o eletrodo de $\mathrm{Ti} / \mathrm{TiO}_{2}$.

Os resultados da Tabela 2, quando comparados com os da Tabela 1, demonstram que, utilizando as condições experimentais aproximadas, as descolorações dos corantes utilizados são estatisticamente iguais ( $p>0,05$ ). Estes dados são fundamentais para ratificar a reprodutibilidade do método.

Tabela 2. Descoloração dos corantes remazol $(0,01 \mathrm{mg} / \mathrm{mL})$ em $\mathrm{KCl}$ $0,1 \mathrm{~mol} \mathrm{~L}^{-1} \mathrm{em} \mathrm{pH} \mathrm{3,35.} \mathrm{Período} \mathrm{de} \mathrm{irradiação:} \mathrm{4,5} \mathrm{h} \mathrm{e} \mathrm{temperatura}$ $30,1 \pm 0,85^{\circ} \mathrm{C}$.

\begin{tabular}{lccc}
\hline Corantes & $\mathrm{m}(\mathrm{mg})$ & $\mathrm{e}(\mu \mathrm{m})$ & $\mathrm{D}(\%)$ \\
\hline Remazol red & $2,35 \pm 0,07$ & $5,60 \pm 0,17$ & $* * 97,73 \pm 3,22$ \\
rb-133 & $(2)$ & $(2)$ & $(2)$ \\
Preto remazol b & $2,20 \pm 0,28$ & $5,24 \pm 0,67$ & $* * 98,97 \pm 0,62$ \\
& $(2)$ & $(2)$ & $(2)$ \\
Alaranjado & $2,40 \pm 0,00$ & $5,71 \pm 0,00$ & $* * 96,05 \pm 0,86$ \\
remazol 3 r & & $(2)$ & $(2)$ \\
Amarelo ouro & $2,15 \pm 0,21$ & $5,12 \pm 0,42$ & $* * 97,57 \pm 2,84$ \\
remazol rnl & $(2)$ & $(2)$ & $(2)$ \\
\hline
\end{tabular}

Obs: $\mathrm{m}=$ massa de $\mathrm{TiO}_{2}$ depositado sobre Ti; e = espessura dos filmes; $\mathrm{D}=$ descoloração. **valores estatisticamente iguais.

\section{Espectros de absorção UV-Vis antes e após a degradação fotoeletroquímica}

Na Figura 1 são apresentados os espectros UV-Vis dos corantes Remazol Red RB-133 e Alaranjado Remazol 3 R, obtidos antes e após a degradação, durante 4,5 h sobre a superfície do eletrodo de Ti/TiO ${ }_{2}$ em solução de $\mathrm{KCl} 0,1 \mathrm{~mol} \mathrm{~L}^{-1}$ ajustada para o $\mathrm{pH} 3,35$ e aplicando-se uma diferença de potencial de $5 \mathrm{~V}$.

Para o espectro do corante Remazol Red RB-133 (Figura 1A) foi observado que antes da fotodegradação o corante apresentou o máximo de absorção em $287 \mathrm{~nm}$, atribuída ao grupo aromático e outra banda na região de $520 \mathrm{~nm}$, característica do grupo azo conjugado à parte cromófora do azocorante por transferência de carga. ${ }^{28}$ Após o processo de fotoeletrocatálise foi observado um completo desaparecimento da banda em $520 \mathrm{~nm}$ e, ainda, a presença de um ombro em torno de $287 \mathrm{~nm}$.

Para os outros azocorantes antes da fotodegradação, os espectros UV-Vis apresentavam máximos de absorção em 252, 296, 386 e 494 nm para o Alaranjado Remazol 3 R (Figura 1B), 309, 390, 481 e 598 nm para o Preto Remazol B (espectro não apresentado) e em 290 e 408 nm, para o Amarelo Ouro Remazol RNL (espectro não apresentado). Após o processo, os espectros das soluções fotodegradadas apresentaram um completo desaparecimento das bandas acima citadas e a presença de ombros entre 281 a $235 \mathrm{~nm}$. No caso específico do Alaranjado Remazol $3 \mathrm{R}$ foi observada uma banda em $274 \mathrm{~nm}$ (Figura 1B). Considerou-se, através destes resultados, que as estruturas dos azocorantes devem estar sofrendo não só o processo de descoloração, mas também o de degradação, podendo formar compostos intermediários.

\section{Espectros de absorção FT-IR antes e após a degradação fotoeletroquímica}

Na Figura 2 são apresentados os espectros FT-IR antes e após a degradação do Remazol Red RB-133 e Amarelo Ouro Remazol RNL, nas condições especificadas.

Considerando previamente a estrutura dos azocorantes estudados, foi constatado que todas são compostas de agrupamento
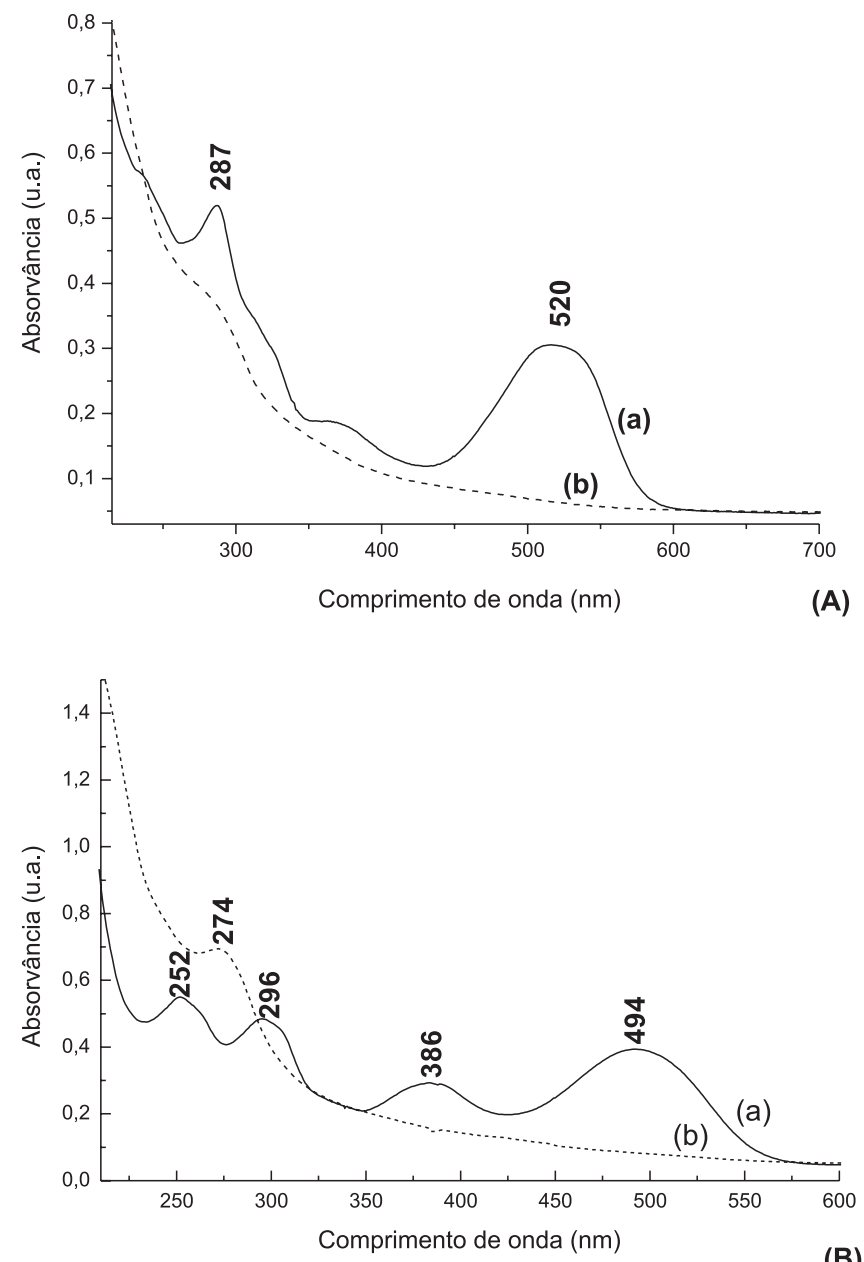

Figura 1. Espectros UV-Vis do (A) Remazol Red RB-133; (B) Alaranjado Remazol $3 R$ obtidos (a) antes e (b) após a degradação durante 4,5 h sobre a superfície do eletrodo de $\mathrm{Ti} / \mathrm{TiO} \mathrm{O}_{2}$ em solução de $\mathrm{KCl}$ 0,1 $\mathrm{mol} \mathrm{L}^{-1}$ em uma diferença de potencial de $5 \mathrm{~V}$. O pH da solução foi 3,35

azo $(\mathrm{N}=\mathrm{N})$ ligado a diferentes grupos aromáticos, apresentando esta frequência característica entre $1415-1411 \mathrm{~cm}^{-1}$. Nos espectros FT-IR das amostras degradadas e, posteriormente, liofilizadas, em comparação com os mesmos espectros das amostras das soluções dos corantes puros e liofilizados, foram observadas bandas intensas entre $1405-1411 \mathrm{~cm}^{-1}$. Na Figura 2 são apresentados apenas os espectros do Remazol Red RB-133 e Amarelo Ouro Remazol RNL, onde se constatam as bandas entre $1405-1407 \mathrm{~cm}^{-1}$, os outros espectros não foram apresentados. Estas bandas podem ser atribuídas ao estiramento $(\mathrm{N}=\mathrm{N})$ dos grupos intermediários remanescentes da degradação. ${ }^{29,30}$ Foi considerado que esta banda esteja relacionada ao grupo azo de compostos aromáticos não simétricos, pois para o grupo azo de compostos aromáticos simétricos, com centro de simetria, esta frequência não é ativa no infravermelho, sendo somente ativa no espectro Raman. ${ }^{31}$ Ocorreu, desta forma, o desaparecimento da maioria das bandas predominantes nos espectros dos azocorantes, confirmando que os diferentes corantes sofreram não somente o processo de descoloração, mas a degradação da sua estrutura original.

Além desta banda característica, foram observadas, nos espectros FT-IR das amostras degradadas, outras frequências centradas entre 1099-1113, 1260-1266 e 1646-1637 cm-1. Para tentar relacionar um determinado composto a estas bandas, foi considerado que existiria semelhança com o espectro FT-IR do ácido sulfanílico $\left(\mathrm{NH}_{2}-\phi-\mathrm{SO}_{3} \mathrm{H}\right)$, 

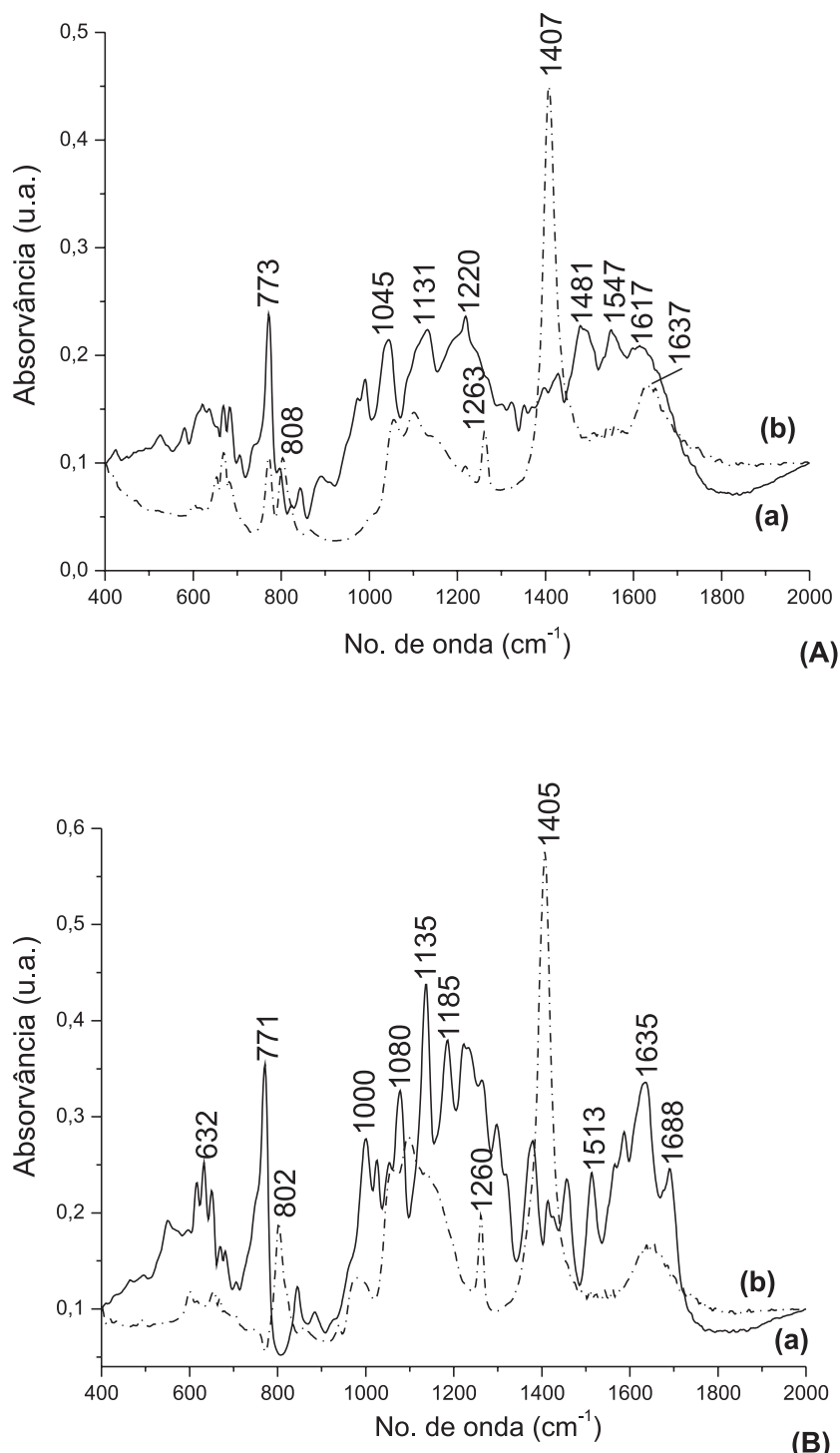

Figura 2. Espectros FT-IR do (A) Remazol Red RB-133 e (B) Amarelo Ouro Remazol RNL obtidos (a) antes e (b) após a degradação durante 4,5 hobre a superfície do eletrodo de Ti/TiO em solução de $\mathrm{KCl}$ 0,1 mol L $\mathrm{L}^{-1}$ em uma diferença de potencial de 5 V. O pH $\cong 3,35$

muito próximo a um provável produto intermediário. O ácido sulfanílico apresenta bandas intensas no FT-IR em 1499, 1317, 1245, 1125, 1167 , 1011 e $1037 \mathrm{~cm}^{-1}$. Desta forma, concluí-se a inviabilidade desta comparação com as bandas observadas das amostras de corantes degradados.

Outra possibilidade seria considerar a formação de compostos cujos grupos azo estariam ligados por estruturas aromáticas, conservando grande parte da estrutura inicial do azocorante. Esta possibilidade foi avaliada pelos trabalhos de Baiocchi et al..$^{32}$ e Prevot et $a l .,{ }^{33}$ pela técnica HPLC/MS, onde foram estudados os fragmentos da estrutura molecular de compostos-modelo de corantes, confirmando que o processo de degradação pode levar à formação de compostos do tipo azobenzeno $(\varnothing-\mathrm{N}=\mathrm{N}-\varnothing)$, sendo que as substituições no anel benzênico seriam conforme o corante degradado. Outra interpretação das frequências observadas no FT-IR para o produto degradado pode ser baseada no trabalho de Galindo et al. ${ }^{34}$ que por GC/MS, HPLC, NMR e FT-IR caracterizaram as espécies formadas durante o processo de oxidação de um composto aminoazobenzeno. Foram relacionadas as bandas em 1420, 1686 e $1350 \mathrm{~cm}^{-1}$ aos compostos de acetato de sódio, oxalato de sódio e nitratos inorgânicos, respec- tivamente. Entretanto, esta caracterização considerou uma rápida decomposição dos produtos intermediários em $\mathrm{CO}_{2}$ e ácidos alifáticos. Mas foi discutido também, pela comprovação com estas técnicas, que na solução degradada ocorrem produtos intermediários, do tipo azobenzeno substituído.

Considerando que os autores destes artigos puderam determinar os diferentes agrupamentos do tipo azobenzeno durante a degradação de compostos muito semelhantes ao estudado neste trabalho e as frequências obtidas das amostras dos azocorantes degradados podem ser relacionadas a estruturas aromáticas,${ }^{31}$ mesmo que exista uma dificuldade de relacionar as bandas obtidas exatamente com estes compostos, os resultados podem ser um indicativo da ocorrência de estruturas intermediárias mais complexas e estáveis em solução.

\section{CONCLUSÃO}

Para os azocorantes estudados, o método tornou-se muito eficaz, atingindo percentuais de descoloração acima de $83 \%$. Esse percentual tornou-se ainda mais elevado quando se variou o potencial (aplicado sobre os eletrodos de $\mathrm{Ti} / \mathrm{TiO}_{2}$ ) de 1 para $5 \mathrm{~V}$, obtendo descolorações com percentuais acima de $96 \%$. Este fato evidenciou que a diferença de potencial aplicado sobre os eletrodos consiste em um importante parâmetro experimental para a fotocatálise. Isso fica ainda mais evidente quando se levaram em conta os resultados obtidos com a espectrofotometria UV-Vis, onde se observou (em todos os casos) o desaparecimento das bandas de absorção características do grupo azo conjugado à parte cromófora dos azocorantes.

Outro dado muito importante obtido nos experimentos foi que a descoloração dos corantes remazois e do corante 2 foram estatisticamente iguais $(\mathrm{p}>0,05)$, quando foi utilizado o mesmo potencial ( 5 $\mathrm{V})$. Isso demonstrou a reprodutibilidade da metodologia empregada, independentemente da estrutura química do corante estudado.

Apesar da alta porcentagem observada na descoloração dos corantes, os espectros FT-IR demonstraram a formação de agrupamentos intermediários, provenientes da quebra de ligação preferencial na estrutura química dos corantes remazois.

Considera-se que uma próxima etapa do trabalho deva ser aumentar o tempo de irradiação e, desta forma (monitorando por FT-IR), deseja-se observar a eliminação destes intermediários, acompanhando, também, o processo da mineralização da solução.

\section{MATERIAL SUPLEMENTAR}

Está disponível em http://quimicanova.sbq.org.br, com acesso livre, na forma de arquivo PDF. Na Figura 1S são apresentadas as estruturas moleculares dos corantes estudados e na Figura $2 \mathrm{~S}$ os Difratogramas de raios-X.

\section{AGRADECIMENTOS}

À Lavanderia Industrial CLAREAR pelo fornecimento dos corantes, ao CNPq/PIBIC e PROPPG/UEL pela bolsa de Iniciação Científica dada a T. N. M. Cervantes, à Fundação Araucária (Protocolo Nº. 8782, Convênio 045/2007) e CNPq (Protocolo No. 2901948874277149 , edital 15/2007) pelo auxílio financeiro ao projeto.

\section{REFERÊNCIAS}

1. Passos, J. A. L.; Pereira, F. A.; Tomich, S.; Water Sci. Technol. 1994, 29 , 105.

2. Beltrame, L. T. C.; Tese de Doutorado, Universidade Federal do Rio Grande do Norte, Brasil, 2006. 
3. Kunz, A.; Zamora, P. P.; Moraes, S.G.; Durán, N.; Quim. Nova 2002, 25,78 .

4. http://www.unicamp.br/unicamp/unicamp_hoje/ju/novembro2003/ ju238pag04.html, acessada em Outubro 2009.

5. Mills, A.; Davies, R. H.; Worsley, D.; Chem. Soc. Rev. 1993, 22, 417.

6. Hoffmann, M. R.; Martin, S. T.; Choi, W. Y.; Bahnemann, D. W.; Chem. Rev. 1995, 95, 69.

7. Catanho, M.; Malpass, G. R. P.; Motheo, A. J.; Quim. Nova 2006, 29, 983.

8. de Santana, H.; Bonancea, C. E.; Takashima, K.; Quim. Nova 2003, $26,807$.

9. Maillard-Dupuy, C.; Guillard, C.; Pichat, P.; New J. Chem. 1994, 18, 941.

10. Nogueira, R. F. P.; Jardim, W. F.; Quim. Nova 1998, 21, 69.

11. Bickley, R. I.; Gonzalez, T. C.; Lees, J. S.; Palmisano, L.; Tilley, R. J. D.; J. Solid State Chem. 1991, 92, 178.

12. Konstantinou, I. K.; Albanis, T. A.; Appl. Catal., B 2004, 49, 1.

13. Liu, G. M.; Wu, T.X.; Zhao, J. C.; Hidaka, H.; Serpone, N.; Environ. Sci. Technol. 1999, 33, 1379.

14. Wu, T. X.; Liu, G. M.; Zhao, J. C.; Hidaka, H.; Serpone, N.; J. Phys. Chem. B 1999, 103, 4862.

15. Matthews, R. W.; J. Phys. Chem. 1988, 92, 6853.

16. Sabate, J.; Anderson, M. A.; Kikkawa, H.; Edwards, M.; Hill, Jr. C. G.; J. Catal. 1991, 127, 167.

17. Bouzaza, A.; Laplanche, A.; J. Photochem. Photobiol., A 2002, 150, 207.

18. Konstantinou, I. K.; Sakkas, V. A.; Albanis, T. A.; Water Res. 2002, 36, 2733.

19. Di Paola, A.; Lopez, G. E.; Ikeda, S.; Marci, G.; Otan B.; Catal. Today 2002, 75, 87 .
20. Palombari, R.; Ranchella, M.; Rol, C.; Sebastian, G. V.; Sol. Energy Mater. Sol. Cells. 2002, 71, 359.

21. Jiang, D.; Zhao, H.; Jia, Z.; Cao, J.; John, R.; J. Photochem. Photobiol., A 2001, 144, 197

22. An, T.; Xiong, Y.; Li, G.; Zha, C.; Zhu, X.; J. Photochem. Photobiol., A 2002, 152, 155

23. de Santana, H.; Macedo, L. C.; Zaia, D. A. M.; Moore, G. J.; J. Photochem. Photobiol., A 2007, 185, 86.

24. Manechini, B. P.; Trabalho de Conclusão de Curso, Universidade Estadual de Londrina, Brasil, 2007.

25. Lipp, L.; Pletcher, D.; Electrochim. Acta 1997, 42, 1091.

26. Al-Kandari, S.; Al-Kandari, H.; Al-Kharafi, F.; Katrib; A.; Appl. Catal., A 2008, 341, 160

27. Conde-Gallardo, A.; Guerrero, M.; Castillo, N.; Soto, A. B.; Fragoso, R.; Cabañas-Moreno, J. G.; Thin Solid Films 2005, 473, 68

28. Sakthivel, S.; Shankar, M. V.; Palanichamy, M.; Arabindoo, B.; Murugesan, V.; J. Photochem. Photobiol., A 2002, 148, 153.

29. Machida, K; Kim, B.; Saito, Y.; Igarashi, K.; Uno, T.; Bull. Chem. Soc. Jpn. 1974, 47, 78.

30. Bassignana, P.; Cogrossi, C.; Tetrahedron 1964, 20, 2361.

31. Colthup, N. B.; Daly, L. H.; Wierly, S. E.; Introduction to Infrared and Raman Spectroscopy, Academic Press: New York, 1964.

32. Baiocchi, C.; Brussino, M. C.; Pramauro, E.; Prevot, A. B.; Palmisano, L.; Marci, G.; Int. J. Mass Spectrom. 2002, 214, 247.

33. Prevot, A B.; Fabbri, D.; Pramauro, E.; Baiocchi, C.; Medana, C.; J. Chromatogr., A 2008, 1202, 145.

34. Galindo, C.; Jacques, P.; Kalt, A.; J. Photochem. Photobiol., A 2000, 130,35 . 


\section{ESTUDO DA FOTOCATÁLISE HETEROGÊNEA SOBRE Ti/TiO ${ }_{2}$ NA DESCOLORAÇÃO DE CORANTES} SINTÉTICOS

Thiago N. M. Cervantes, Dimas A. M. Zaia e Henrique de Santana*

Departamento de Química, Universidade Estadual de Londrina, 86051-990 Londrina - PR, Brasil
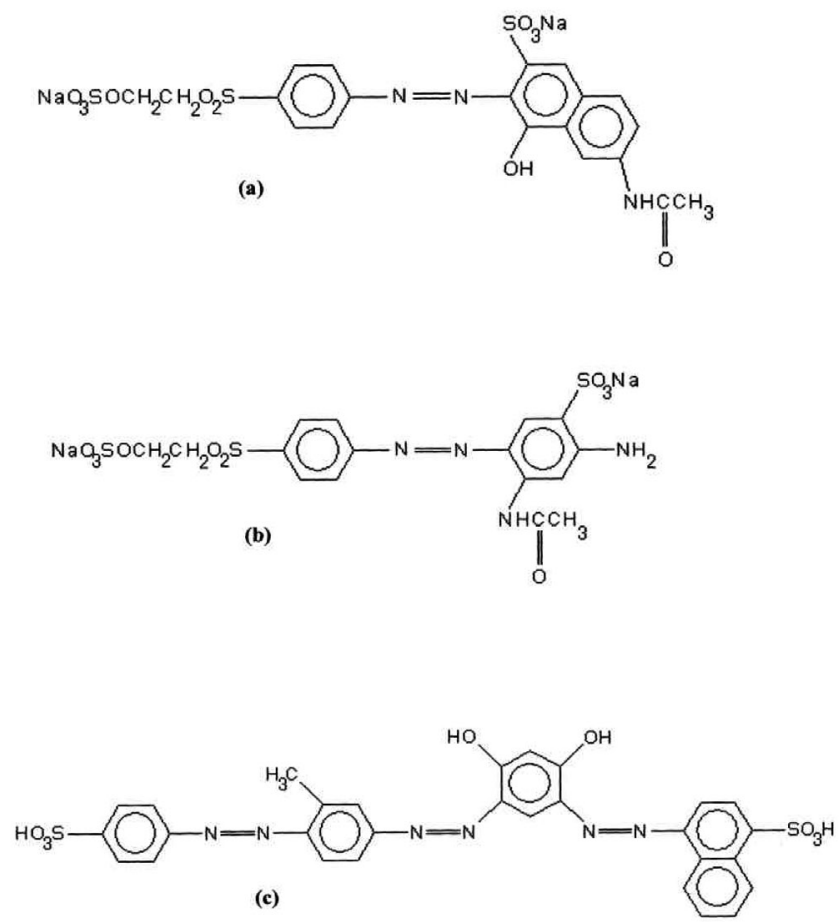

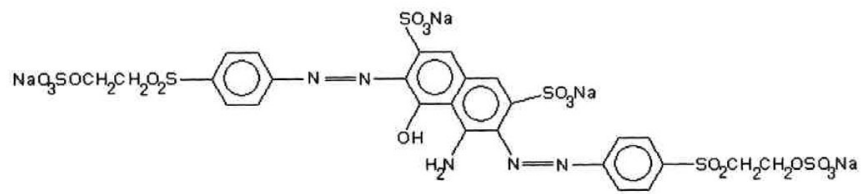

(d)

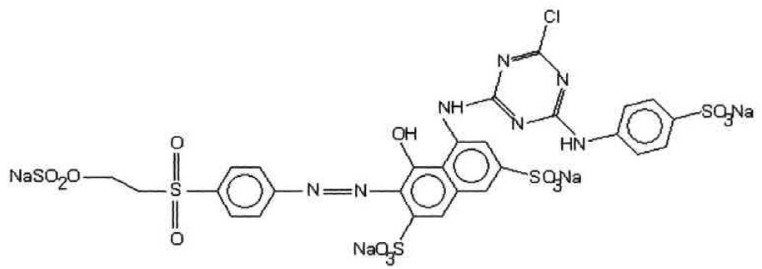

(e)

Figura 1S. Estruturas moleculares dos corantes: (a) Alaranjado Remazol 3 R; (b) Amarelo Ouro Remazol RNL; (c) Azocorante 2; (d) Preto Remazol B; (e) Remazol Red RB-133

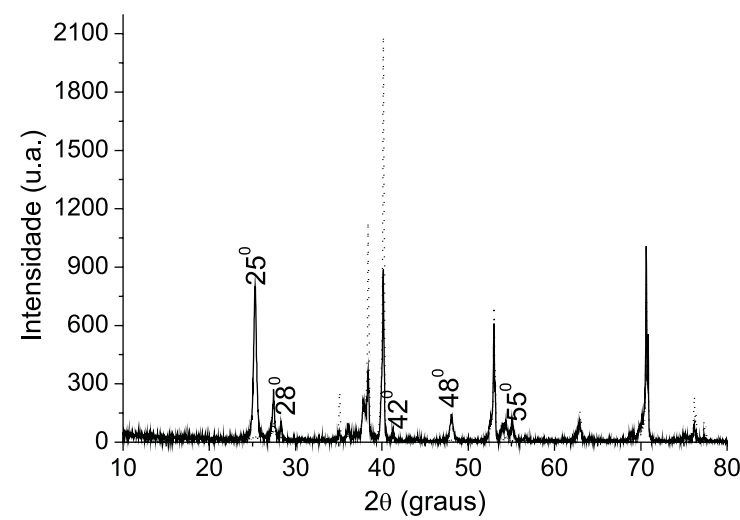

Figura 2S. Difratogramas de raios-X da placa de titânio (-.........) e do filme particulado de $\mathrm{TiO}_{2}$ sobre a placa de titânio (-)

*e-mail: hensan@uel.br 